\title{
Reliable Acquisition of Electroencephalography Data during Simultaneous Electroencephalography and Functional MRI
}

\author{
Hui Ming Khoo ${ }^{1}$, Yuya Fujita ${ }^{1}$, Naoki Tani ${ }^{1}$, Tetsuya Shimokawa ${ }^{2}$, Natalja Zazubovits ${ }^{3}$, Satoru Oshino ${ }^{1}$, Jean \\ Gotman $^{3}$, Haruhiko Kishima ${ }^{1}$ \\ ${ }^{1}$ Department of Neurosurgery, Osaka University Graduate School of Medicine ${ }^{2}$ Center for Information and Neural Networks, National Institute of \\ Information and Communications Technology ${ }^{3}$ Montreal Neurological Institute and Hospital, McGill University
}

\section{Corresponding Author}

Hui Ming Khoo

hui.ming.khoo@mail.mcgill.ca

\section{Citation}

Khoo, H.M., Fujita, Y., Tani, N., Shimokawa, T., Zazubovits, N., Oshino, S., Gotman, J., Kishima, H. Reliable Acquisition of Electroencephalography Data during Simultaneous Electroencephalography and Functional MRI. J. Vis. Exp. (169), e62247, doi:10.3791/62247 (2021).

\section{Date Published}

March 19, 2021

\section{DOI}

$10.3791 / 62247$

URL

jove.com/video/62247

\section{Abstract}

Simultaneous electroencephalography (EEG) and functional magnetic resonance imaging (fMRI), EEG-fMRI, combines the complementary properties of scalp EEG (good temporal resolution) and fMRI (good spatial resolution) to measure neuronal activity during an electrographic event, through hemodynamic responses known as blood-oxygen-level-dependent (BOLD) changes. It is a non-invasive research tool that is utilized in neuroscience research and is highly beneficial to the clinical community, especially for the management of neurological diseases, provided that proper equipment and protocols are administered during data acquisition. Although recording EEG-fMRI is apparently straightforward, the correct preparation, especially in placing and securing the electrodes, is not only important for safety but is also critical in ensuring the reliability and analyzability of the EEG data obtained. This is also the most experience-demanding part of the preparation. To address these issues, a straightforward protocol that ensures data quality was developed. This article provides a step-by-step guide for acquiring reliable EEG data during EEG-fMRI using this protocol that utilizes readily available medical products. The presented protocol can be adapted to different applications of EEG-fMRI in research and clinical settings, and may be beneficial to both inexperienced and expert operators.

\section{Introduction}

Functional magnetic resonance imaging (fMRI) provides a measure of neuronal activity through hemodynamic responses by measuring blood-oxygen-level-dependent (BOLD) changes during an electrographic event.
Simultaneous electroencephalography (EEG) and fMRI (EEG-fMRI) is a non-invasive research tool that combines the synergic properties of scalp EEG (good temporal resolution) and fMRI (good spatial resolution), allowing better localization 
of the site responsible for the generation of electrographic events detectable in EEG. It was first developed in the 1990s for the use in the epilepsy field ${ }^{1,2}$ and has subsequently been used in neuroscience research since the $2000 \mathrm{~s}^{3,4}$. With the increase in knowledge regarding the safety ${ }^{5}$ and continuous development of techniques for the removal of MRI-induced artifacts on $\mathrm{EEG}^{3,6,7,8,9,10}$, it is currently a tool that is widely utilized in both neuroscience and clinical research ${ }^{11}$.

EEG-fMRI is acquired either at rest or during a task, depending on the research question. In general, resting state acquisition allows the identification of structures involved in the generation of a particular EEG feature (e.g., waveform, rhythm, frequencies, power) and helps in understanding the variable spontaneous brain activities ${ }^{11}$. A number of neuroscience studies and most clinical studies, especially those on epilepsy ${ }^{12}$, acquire EEG-fMRI at rest ${ }^{11}$. Task-based acquisition allows the identification of cerebral areas and the brain electrical activities assigned or related to a specific task and helps establish the link between the electrical activities and cerebral areas associated with the task. Task-based acquisition is mainly utilized in neuroscience studies $^{11}$ and some clinical studies ${ }^{13}$. Most task-based EEG-fMRI acquisitions use an event-related design. The type of modeling used for integrating EEG and $\mathrm{fMRI}$ data determines whether the efficiency or detection power should be maximized in designing the task ${ }^{14}$. Please see the studies by Menon et al. ${ }^{14}$ and Liu et al. ${ }^{15,16}$ for details on the task design.

Although data acquisition during EEG-fMRI may appear straightforward, the preparation is experience-demanding. A protocol for guiding proper preparation for data acquisition is important to ensure both the safety and yield (i.e., analyzable and reliable data). Despite the existence of various techniques to remove MRI-induced EEG artifacts, inconsistent artifacts in the EEG recorded, especially those related to machinery-induced vibration of the wires and subjects' gross movements, are still difficult to completely remove; therefore, these artifacts need to be minimized during data acquisition.

This article presents a straightforward protocol that utilizes readily available MRI-compatible medical products. The protocol provides important steps that ensure data quality, particularly the quality of EEG data, which is key to the success of an EEG-fMRI study. This protocol was developed based on the 20-year experience of the EEG-fMRI research team at the Montreal Neurological Institute ${ }^{12,17}$ and was further modified for use at Osaka University, which benefits both inexperienced and expert operators.

\section{Protocol}

The research ethics committee of Osaka University Hospital and the safety committee of the Center for Information and Neural Networks (CiNET) approved the protocol (Osaka University Hospital Approval Nos. 18265 and 19259; CiNET Approval Nos. 2002210020 and 2002120020). All subjects provided written informed consent for their participation.

\section{Preparation of the experimental setup}

1. Connect the MRI-compatible EEG and bipolar amplifiers to the battery packs (ensure that they are fully charged) and to the recording computer.

2. Ensure that the workspace of the recording software is correctly set up. Set the amplitude resolution to $0.5 \mu \mathrm{V}$ to avoid amplifier saturation; set the frequency filters according to the frequency band of interest. Set the sampling rate at $5,000 \mathrm{~Hz}$ (maximum possible for 
the amplifiers used in this protocol), regardless of the frequency band of interest.

NOTE: Amplitude resolution at $0.5 \mu \mathrm{V}$ corresponds to a maximum value of $16.38 \mathrm{mV}$, which is sufficient to record the gradient artifact, considering that gradient artifact peaks can reach amplitudes over 100 -fold more than those of spontaneous scalp EEG (approximately 10-100 $\mu \mathrm{V})$ at high speeds $(>1,000$-fold faster than the rate of change of ongoing EEG). Theoretically, the sampling rate should be at least twice as high (Nyquist theorem) as the highest frequency in the gradient switching spectrum, in order to accurately sample the highfrequency gradient-switching artifacts and detect the true onset of each volume's gradient activity for subsequent removal $^{12,18}$. However, increasing the sample rate results in large file sizes, which require significant investment for data storage and may also impede subsequent post-processing. Using the syncing device makes it unnecessary to raise the sample rate to improve synchronization between the EEG and MR clocks (see step 1.4). A sampling rate of $5,000 \mathrm{~Hz}$ is adequate for usual EEG/event-related potential (ERP) recordings, and higher sample rates do not improve data quality because the subsequent artifact correction process, which involves down sampling of the data to a frequency below $500 \mathrm{~Hz}$ and additional low-pass filtering, eliminates all high-frequency gradient correction residuals that may exist $^{18}$.

3. Refer to the manual for details regarding the proper settings of the recording software needed for EEG acquisition in the MRI, which differs from that outside the MRI.

4. Check whether markers from the scanner, i.e., the markers for clock synchronization (sync on by default) and volume trigger (R128 by default), are periodically displayed in the online EEG recording. The sync on display indicates that the MRI scanner and EEG clocks are synchronized, and R128 indicates that the volume triggers are recorded for subsequent post-processing. The MRI scanner and EEG clocks are synchronized using the SyncBox device, which detects the scanner clock output (usually $10 \mathrm{MHz}$ and above), downsamples, and outputs the clock signal (and the synchronization markers) to the USB2 interface.

NOTE: The USB2 interface sends the EEG data from all of the amplifiers, which are phase locked to the scanner clock signal, to the recording computer ${ }^{18}$. Periodic sync on markers are triggers generated from the scanner electrical pulse to synchronize the EEG signal sampling by the MR scanner rate, a requisite for scanner artifact correction. Volume triggers are used to identify MR volume scan onset time for scanner artifact correction during offline EEG processing ${ }^{19}$.

5. Set up the MRI scanner according to the need and availability. It is best to use a transmit and receive head radio frequency $(\mathrm{RF})$-coil to minimize the risk of RF heating. However, a whole body transmit RF-coil and a 20-channel head receive only RF-coil were used here because a transmit and receive head coil was not available for the scanner used (typically the case for most modern scanners).

6. Load a $10 \mathrm{~mL}$ syringe (or several as needed) with the abrasive conductive gel for application of the EEG cap. One could preload the abrasive gel in a $50 \mathrm{~mL}$ largecapacity plastic syringe for fluid dispensing and fill the 10 $\mathrm{mL}$ syringe with the gel prior to the subject's arrival.

NOTE: Application of a 32-channel EEG cap typically consumes about $20-25 \mathrm{~mL}$ of gel. 


\section{Applying the EEG cap and ECG electrode}

1. At recruitment, ask the subject to complete a checklist of potential contraindications for MRI. Confirm that the subject has no contraindications for MRI prior to arrival. NOTE: In general, any subject who qualifies for MRI can participate in an EEG-fMRI study. The exclusion criteria are: non-cooperative or non-compliant subjects; those with underlying conditions (e.g., chronic back pain), which prevent them from lying supine for a certain period of time (typically at least $1 \mathrm{~h}$ ); or subjects who may be unable to lie still on the MRI table during the scan. Movement not only hampers the quality of both EEG and $\mathrm{fMRI}$ data but also imposes a potential hazard to the subjects themselves (e.g., induces current in the wires and cables that may cause stimulation). In the case of task-based acquisition, the language comprehension ability of the subject should also be considered (avoid subjects who are unable to understand the instructions). In this study, 32 healthy volunteers (mean age, 40 years; 17 women) and 25 patients with epilepsy (mean age, 31 years; 13 women) were recruited.

2. Ask the subjects to wash their hair with shampoo without conditioner or wax before arrival.

3. Explain the purpose of the experiment and next steps to the subject.

4. Measure the head circumference (i.e., occipital frontal circumference) by wrapping a flexible non-stretchable measuring tape around the head over the supraorbital ridges and the occiput and select an appropriately sized cap. Use a cap that is $1 \mathrm{~cm}$ larger than the head circumference, and always ask the subject whether the cap is comfortable once placed (i.e., not too tight).
5. After placing the cap at the approximate position over the subject's head, using the same measuring tape, measure the lengths of the inion-nasion arc, defined as the arc over the midline of the head extending from the occiput to the bridge of the nose, and the peri-auricular arc, defined as the arc extending between the ears that crosses the midpoint of inion-nasion arc, over the cap. Mark the intersection of the inion-nasion arc and the peri-auricular arc (the point where the midpoints of both arcs meet, AKA $\mathrm{Cz}$ ), and slide the cap over the head so that the position of electrode $\mathrm{Cz}$ is adjusted to this intersection. Ensure that the cap is not rotated horizontally by manually checking whether electrodes Fz, Pz, Oz, Reference, and Ground are positioned over the inion-nasion arc.

6. Expose the skin underneath each electrode by displacing the hair to the side of the electrode using the back of a cotton swab.

7. Rub the skin beneath each electrode by quickly spinning a cotton swab containing $70 \%$ alcohol solution placed through the opening of the electrode.

8. Apply a small amount of the abrasive conductive gel $(\sim 0.2 \mathrm{~mL})$ in the opening and abrade the skin by quickly spinning a cotton swab in a similar fashion.

9. Monitor the impedance of the electrode (displayed by the recording software) and repeat the abrasion as stated in step 2.8 until the impedance drops at least below 20 $\mathrm{k} \Omega^{20}$, preferably as low as possible (below $\left.5 \mathrm{k} \Omega\right)^{21}$.

10. Fill the opening with the same gel (usually $\sim 0.5 \mathrm{~mL}$ ) once the impedance is satisfactory. Do not apply excessive gel in the opening to avoid bridging between electrodes. Move to the next electrode if the impedance is unsatisfactory despite repeated abrasion and come 
back later because sometimes the impedance continues to drop with time after applying the gel.

11. Repeat steps 2.6-2.9 for all of the scalp EEG electrodes.

12. Before placing the ECG electrode at the back, ask the subject to sit upright without flexing the neck.

13. Make sure that the ECG electrode wire is straight when placing the ECG electrode at the back but keep some allowance for laying the ECG electrode wire along the curve of the neck, to avoid displacement of the electrode when the subject lays down on the MRI table. Place the ECG electrode $2-3 \mathrm{~cm}$ left from the median furrow, which can be identified as the vertical indentation along the midline of the back. The vertical position varies depending on the subject's height; it is typically positioned on the lower back approximately on the line that extends between the tips of the scapula in a subject of about $160 \mathrm{~cm}$.

14. Rub the skin underneath the ECG electrode with an alcohol swab.

15. Attach the ECG electrode to the skin using a doublesided adhesive ring and repeat steps 2.8-2.9. The adhesive ring also serves as a padding to avoid direct contact of the electrode with the skin.

16. Fold the dry alcohol cotton swab into four, and place it on the ECG electrode. Tape it to the skin using a surgical tape (medical adhesive tape). Tape the ECG electrode wire to the skin up to the shoulder.

\section{Apply the carbon wire loop (if a bipolar amplifier is available)}

1. Place a set of pre-braid carbon wire (diameter $1 \mathrm{~mm})^{9}$ consisting of six loops (diameter $10 \mathrm{~cm}$ ) over the cap in a position such that the bundle of the wires come in parallel with the bundle of the electrodes on the top of the head.

2. Use surgical tape $(1 \times 2 \mathrm{~cm})$ to secure the loops around the electrodes, so that the loops cover the head with each loop covering evenly almost an equal area (i.e., both the fronto-temporal, both the temporo-occipital, the occipital, and the vertex). Alternatively, one could also sew the loops to the EEG-cap, if applicable.

NOTE: The carbon wire loops on the head serve to capture movement, including ballistocardiogram (BCG). These signals are used for the removal of BCG artifacts from the EEG during offline EEG processing ${ }^{9}$.

\section{Securing the cap and carbon wire loops}

1. Make sure that the EEG electrodes are not forming loops.

2. Wrap the subject's head with an elastic bandage over the EEG cap and the carbon loops. The bandage serves to press the EEG electrode tightly onto the skin, to reduce MRI machinery-induced vibration of the electrodes and prevent the gel from spilling onto the pillow when placing the subject inside the MR scanner (see step 5).

3. Make sure that the bandage covers all of the electrodes and is not too tight by asking whether the subject feels uncomfortable pressure on the head while applying the bandage.

\section{Placing the subject in the MR scanner}

1. In the case of resting-state acquisition, instruct the subject to apply MRI-compatible earbuds in the ears. In the case of task-based acquisition, instruct the subject to apply the MRI-compatible headset or earphones as per the requirement of the experiment. Make sure that the 
subject can hear through both sides of the headset or earphones.

2. Place an MRI-compatible flat memory foam pillow in the lower half of the head coil before asking the subject to lie down and place the head in the coil.

3. After positioning the head appropriately (top of the head placed as close as possible to the top of the head coil), place the electrode and carbon wire bundles straight through the top opening of the head coil.

4. Add memory foam pillows to the top of the head, forehead, and temporal area. The pillows should appropriately fill up all spaces left within the head coil while not compressing the subject's head too tightly.

1. Make sure that the pillows are not squeezing the head while placing the upper half of the head coil and while closing the coil. Adjust the pillows or change to smaller size pillows if too tight. In this way, the pillows serve to hold the electrode wires to reduce MRI machinery-induced vibration on the electrode wires and to restrain head movements while maintaining the comfort of the subject during the scan.

2. Place a half cylinder-shape memory foam pillow at the back of the neck so that the ECG electrode wire is sandwiched well between the pillow and the neck. The portion of ECG electrode wire that passes at the back below the shoulder is indeed sandwiched between the back of subject and the MRI table and is thus immobilized by the subject's own weight.

5. In the case of a task-based acquisition, after placing all of the memory foam pillows, make sure that the headset or earphones are not displaced by testing again if the subject can still hear through both sides of the headset or earphones. After closing the head coil, place the mirror and instruct the subject to adjust the mirror (in the case of the task that requires visual stimuli). Instruct the subject to adjust the mirror if necessary, after moving the table in order to place the subject's head at the isocenter of the MRI bore.

6. Connect the amplifiers placed at the back of the MRI bore to the recording computer placed in the console room using the optic fibers provided.

7. After connecting the EEG/ECG electrodes and the carbon wire loops to the EEG and the bipolar amplifiers at the back of the MRI bore, switch on the amplifiers. Again, check the impedance of all electrodes to make sure that they are still low (at least below $20 \mathrm{k} \Omega$ ). Remove the subject from the MR scanner for adjustment if there is any electrode with high impedance.

\section{Configuration of the wires and amplifiers}

1. Arrange all of the wires between the outlet of the top opening of the head coil and the amplifiers (including the electrodes and carbon wire bundles, the connector box, and the ribbon wires) so that they are placed straight and at the center of the MRI bore. This is important to minimize MRI-induced current.

2. Place one carbon wire loop around the ribbon cable going from the EEG/ECG electrodes connector box to the amplifier and connect all of the carbon wire loops (see step 5.7) to the input box of the bipolar amplifier (EXG MR). This loop serves mainly to capture the vibrations caused by the Helium pump ${ }^{9}$.

3. To minimize MRI machinery-induced vibration, immobilize the wires by sandwiching all of them with MR-safe and non-ferromagnetic sandbags all along the way between the outlet of the top opening of the head 
coil and the amplifiers. Also, place sandbags on the amplifiers. These sandbags, measured $330 \mathrm{~mm} \times 240$ $\mathrm{mm} \times 50 \mathrm{~mm}$ and weighing $4 \mathrm{~kg}$, are supplied by the EEG manufacturer.

4. Position the amplifiers outside the bore of the magnet, which is allowed by the length of the cables as supplied by the manufacturer.

\section{EEG-fMRI data acquisition}

1. Make sure that the subject is comfortable with the positioning before leaving the scanner room, to avoid unnecessary subject movement during the acquisition. Instruct the subject to press the alarm button if necessary (i.e., in case of emergency or if the subject feels an uncomfortable sensation). Communicate with the subject from the console room to confirm that the subject can hear the operator. Tell the subject that loud noises are expected during data acquisition. Instruct the subject as required for the experiment, and instruct the subject not to move during data acquisition.

2. Start the EEG recording before starting $f M R I$ acquisition. Typically, the following images are acquired sequentially: scout images (two-dimensional) for positioning the fMRI field of view, fMRI, and structural images for coregistering the $\mathrm{fMRI}$ images during postprocessing. Shim sequences were run prior to acquiring each type of image for calibration of appropriate parameters.

NOTE: It is important to use MRI sequences that are proven safe with amplifiers to maintain safety, and to avoid any damage to the amplifiers ${ }^{18}$. Details regarding the sequences considered safe will not be discussed in detail. Readers are encouraged to consult the user manual or the support team. In general, gradient echo sequences are recommended and spin echo sequences or any sequence with equivalent RF emission parameters, which can cause excessive RF-induced heating, should be avoided. Heating can be indirectly quantified using metrics that measure the amount of RF exposure, such as specific energy absorption rate $(S A R)$ and the root mean square value of $B_{1}+$ averaged over $10 \mathrm{~s}$ (B1+rms). Recently, $\mathrm{B}_{1}+\mathrm{rms}$, dependent on the imaging parameters but independent of the subjects' body mass ${ }^{22}$, is becoming the new standard to specify the limit. For example, the $\mathrm{B}_{1}+\mathrm{rms}$ thresholds for acquisition at $3 \mathrm{~T}$ using the Brain Products EEG cap are $1 \mu \mathrm{T}$ for the current standard cap and $1.5 \mu \mathrm{T}$ for the new standard EEG cap with a shorter $(10 \mathrm{~cm})$ bundled cable $^{23}$. Flip angle, number of slices, and repetition time (TR) are parameters that need to be considered to keep SAR and $B_{1}+$ rms low. A small flip angle $\left(<90^{\circ}\right)$ is recommended. The number of slices and TR can be adjusted as long as the resulting sequence is below the threshold of $B_{1}+r m s^{23}$.

3. Upon starting the acquisition, make sure again markers from the scanner (see 1.4) are periodically displayed in the online EEG recording.

\section{Representative Results}

Upon placing the EEG cap using this protocol, the impedance of each electrode usually drops below $20 \mathrm{k} \Omega$ (Figure 1). Representative EEG signals obtained from a subject (20year-old man) who participated in a neurocognitive study, and a different subject (19-year-old woman) who participated in an epilepsy study using this protocol in the same MR scanner are shown in Figure 2 and Figure 3, respectively. The subject who underwent neurocognitive testing was instructed to keep the eyes open but stay still while performing a visual task as 
instructed. The subject for the epilepsy study was instructed to close the eyes and sleep, as epileptic activities are typically more frequent during sleep. The EEG signals acquired from both studies were similar before processing (Figure 2); the MRI gradient artifact obscured the real EEG signals. The EEG signals from both studies were processed offline as follows: MRI artifacts were removed using the subtraction method $^{24}$; and BCG, movements, and Helium pump artifacts were removed using the regression of signals recorded from the carbon wire loops ${ }^{7,9}$. The resultant EEG signals (Figure 3B) from both studies were of analyzable quality without visible contamination of BCG artifacts (Figure 3A).
Epileptic activities were clearly seen on the EEG during the epilepsy study (Figure 3B). On the EEG acquired during the neurocognitive study, blinking, eye movement, and muscle artifacts were seen, especially in the frontal leads (Fp1 and Fp2) after artifact removal (Figure 3B) due to the nature of the study, and may be further removed using other methods depending on the need. No artifact originating from machinery vibrations was seen on post-processed EEG signals acquired during both studies (Figure 3B comparable to EEG signals acquired outside MRI as shown in Figure 3C). No artifact originating from the EEG electrodes was seen on the MR images acquired simultaneously (Figure 4).

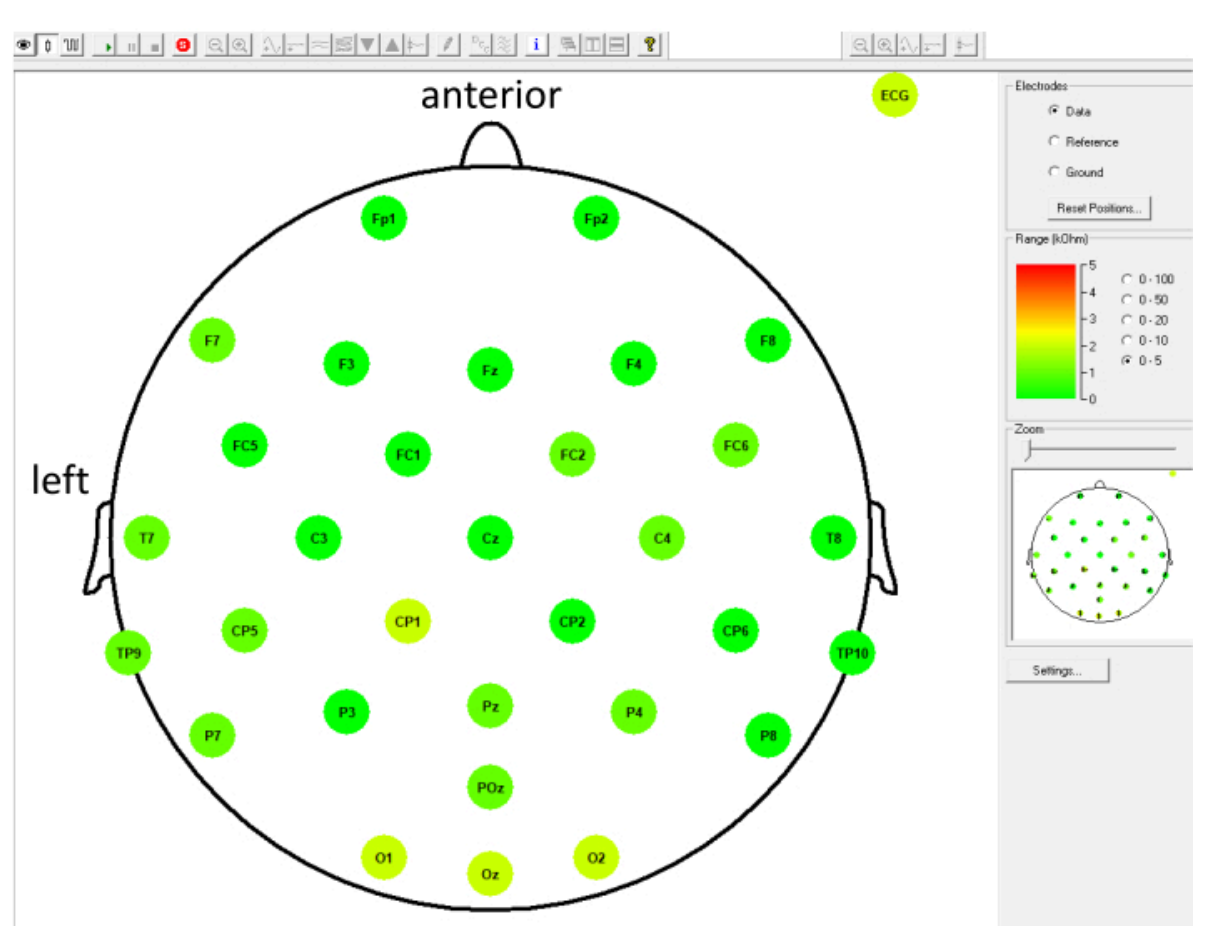

Figure 1: Representative EEG electrodes impedance that dropped below $5 \mathrm{k} \Omega$ upon application of a 32-channel EEG cap on a subject who participated in a neurocognitive study. Each round colored circle represents an EEG electrode, with the electrode name written within the circle; the position of each circle represents the position of each electrode on the EEG cap. The color bar and the numbers on the right represent the range of the impedance being measured $(0-5 \mathrm{k} \Omega$ in this 


\section{jove}

case); green color indicates that the impedance value is lower than the Good level value, and red color indicates Bad level. In this example, electrodes CP1, O1, Oz, O2, and ECG are indicated in light green, which means that the impedances of these electrodes were $2 \mathrm{k} \Omega$; the rest of the electrodes are indicated in dark green, which means that the impedances of these electrodes were $0 \mathrm{k} \Omega$. Please click here to view a larger version of this figure.

\section{Raw EEG signal}

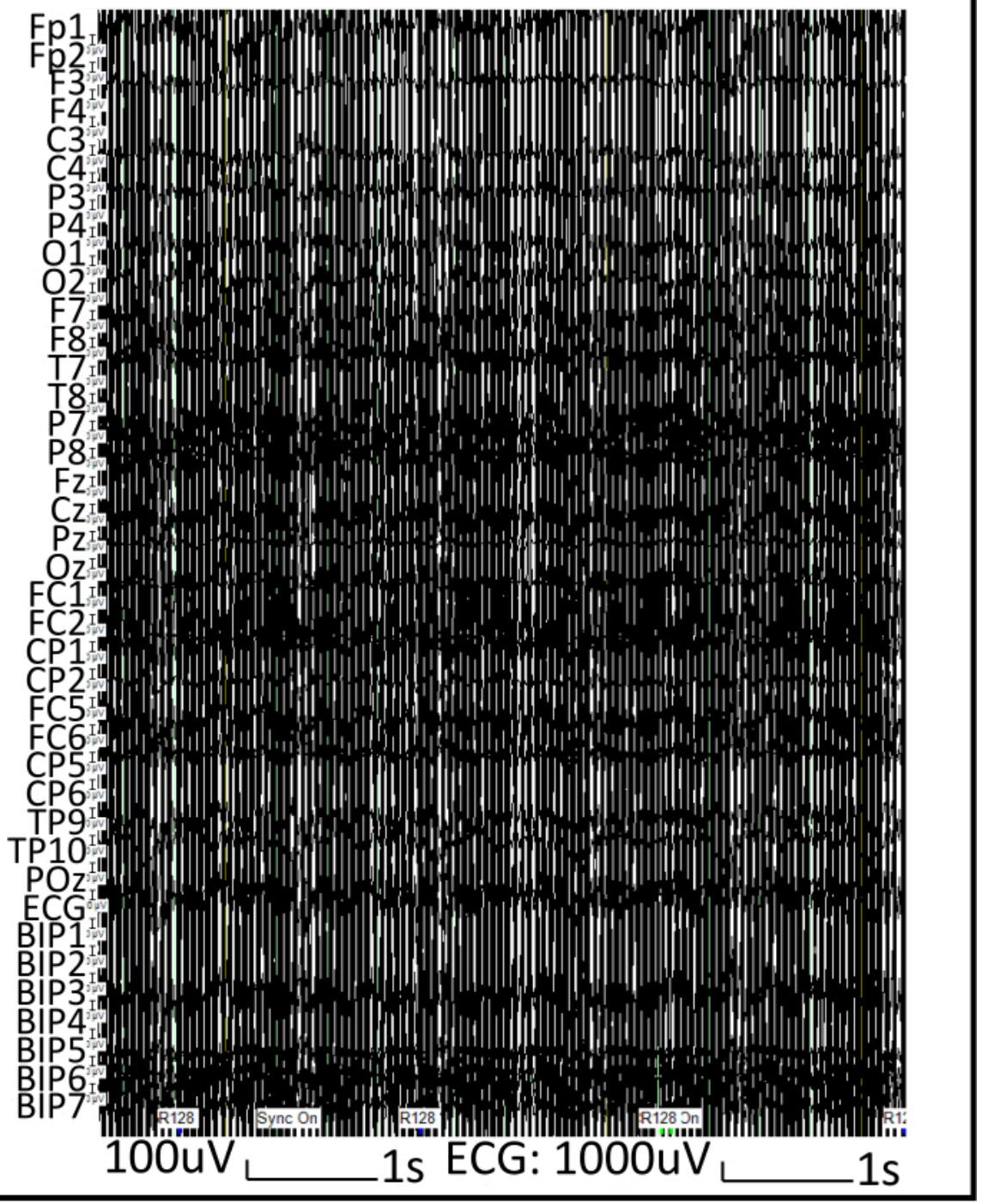

Figure 2: EEG signal before processing. Note that the MRI gradient artifact obscured the real EEG signals. Please click here to view a larger version of this figure. 


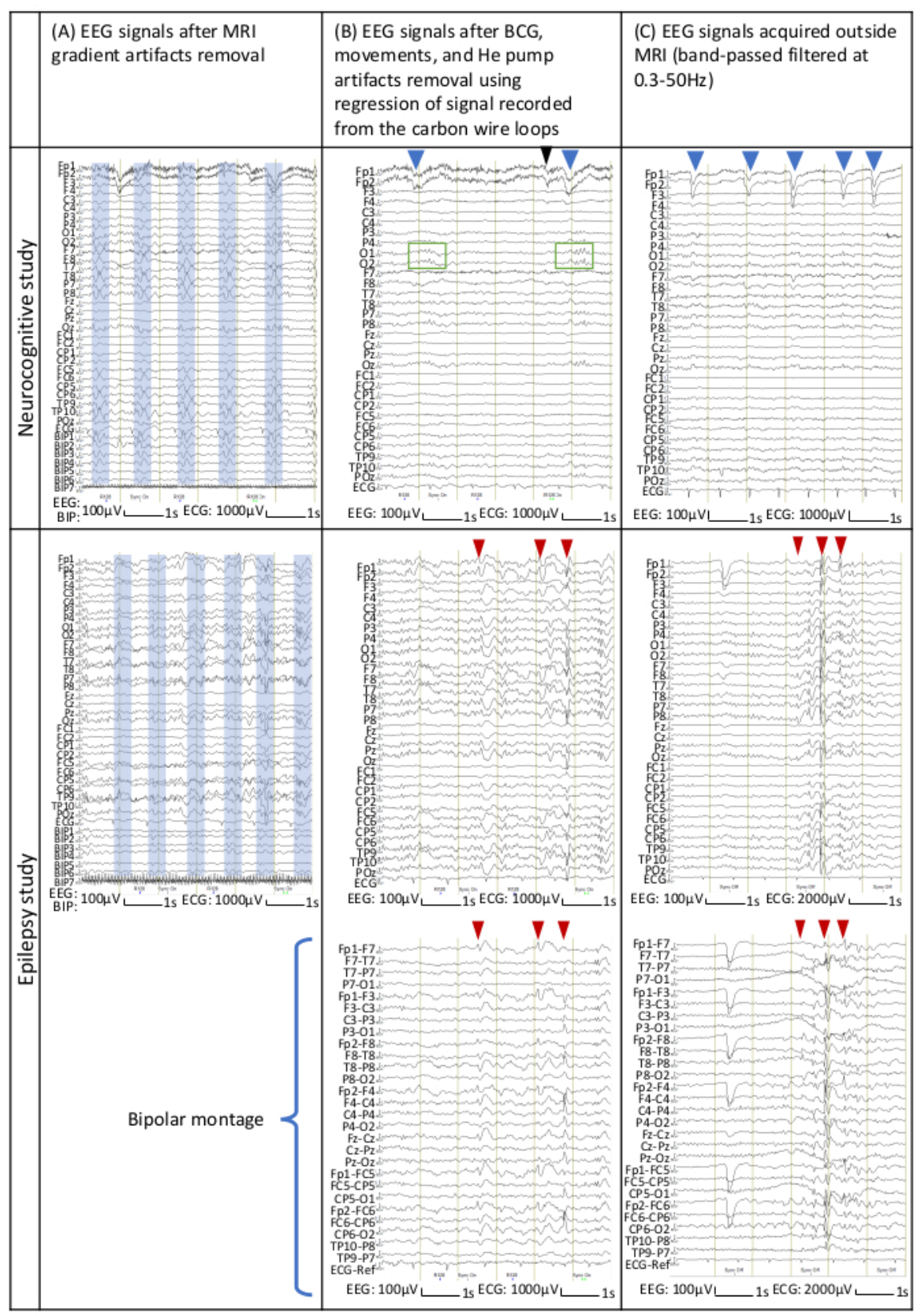

Figure 3: Representative EEG signals from subjects who participated in neurocognitive and epilepsy studies. EEG signals on the top row were from a neurocognitive study and those on the bottom row were from an epilepsy study. EEG signals were processed offline. (A) EEG signals after MRI gradient artifact removal. The boxes in light blue indicate BCG artifacts. (B) EEG signals after artifact removal using regression of signals recorded from the carbon wire loops. (C) EEG signals recorded outside MRI using the same EEG equipment. EEG signals were shown in referential montage (reference at FCz); EEG in bipolar montage (each channel represents the voltage difference between a pair of adjacent electrodes) of the same segment is also shown for EEG acquired during an epilepsy study to ease the visualization of epileptic activities. The 
blue arrowheads ( $\mathrm{B}$ and $\mathrm{C}$, top row) indicate blinking (high-amplitude slow downward deflections/diphasic potentials at Fp1 and Fp2), the black arrowhead (B, top row) indicates eye movement resulting from a saccade or a spontaneous change of gaze (small, rapid deflections at $\mathrm{Fp} 1$ and $\mathrm{Fp} 2$ ), and the green rectangles (B, top row) indicate alpha rhythm seen on the EEG acquired during a neurocognitive study. The low-amplitude and high-frequency activities predominantly at Fp1 and Fp2 are muscle artifacts (thickening of the EEG tracing, top row). The red arrowheads (B and $C$, bottom row) indicate the time points at which epileptic activities were identified on EEG acquired during an epilepsy study (sharp downward or upward deflections that are sometimes followed by a slow wave). Please click here to view a larger version of this figure. 


\section{(A)}

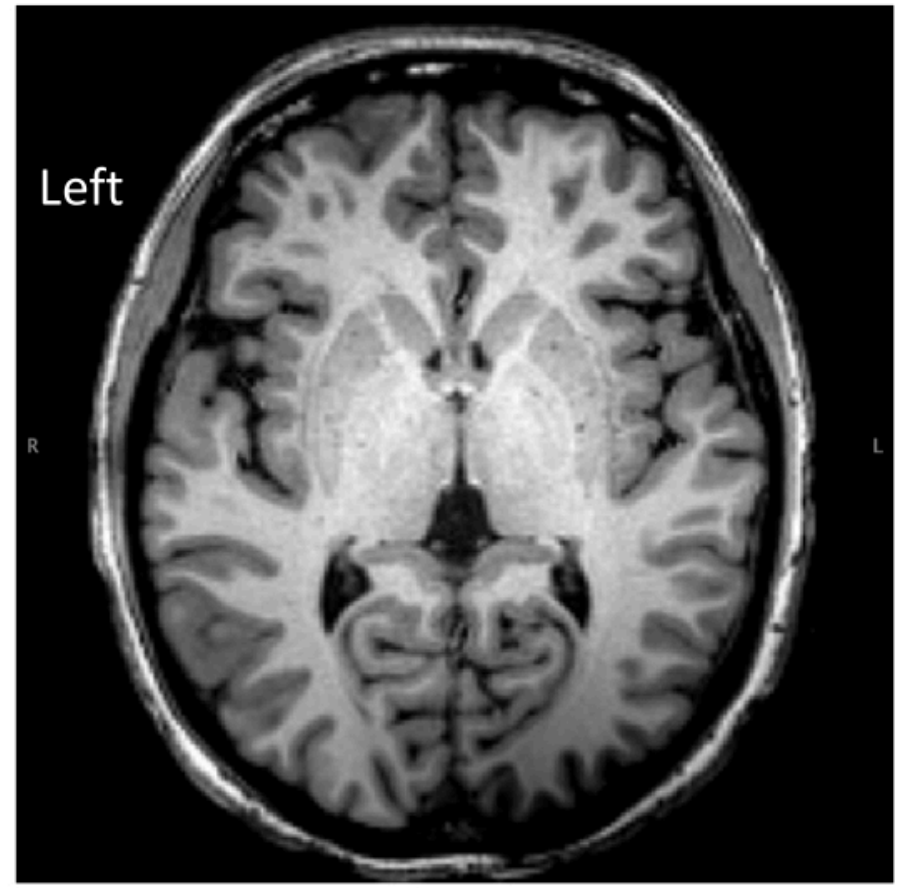

(B)

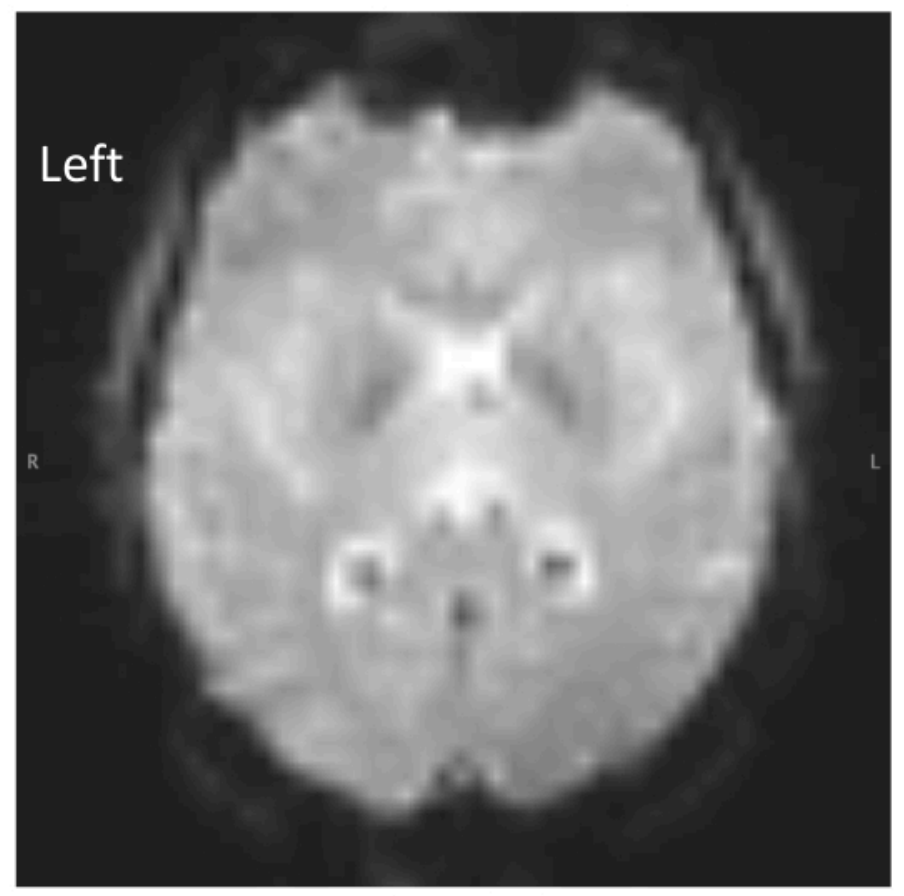

Figure 4: Representative MRI data acquired from a subject using this protocol. Note that the EEG electrodes did not cause visible artifacts on the MR images acquired simultaneously. (A) magnetization prepared rapid acquisition with gradient echo image; (B) echo planar imaging. Please click here to view a larger version of this figure. 


\section{Discussion}

This protocol highlighted the important points for the safe simultaneous EEG-fMRI acquisition of good quality data.

Some common errors resulting in difficult-to-remove artifacts on EEG as well as troubleshooting techniques are as follows. First, choosing subjects that are compliant and cooperative and ensuring their comfort during data acquisition can prevent premature termination due to subject movements (steps 2.1 and 5.4). Second, impedance not dropping below $20 \mathrm{k} \Omega$ after repeated abrasion of the scalp (step 2.9) is most likely due to inadequate brushing after use. Thoroughly brushing each opening of the EEG electrodes when washing the cap prevents this problem. Third, inappropriate settings of the hardware and software can result in saturation of the EEG signals that subsequently hamper artifact removal during offline EEG processing. Lastly, to prevent the recording of saturated EEG signals, maintain the impedance of each electrode below $20 \mathrm{k} \Omega$ after placing the subject in the MR scanner prior to data acquisition; adequately diminish mechanical vibrations by immobilizing the EEG cap (which also means the subject's head), cables and wires; monitor the raw EEG signal online with the recording software and make sure that the sampling rate and amplitude resolution are correctly set up.

The simultaneous acquisition of EEG and $\mathrm{fMRI}$ raises important safety issues related to RF-induced heating and switching gradient-induced currents due to the presence of electrical wires connected to the subject in the rapidly changing magnetic field ${ }^{5}$. These safety issues have been largely minimized over the years following research findings that have enhanced knowledge of this aspect and led to large improvements in the technology of MRI-compatible EEG equipment. Nevertheless, careless preparation without adequate knowledge or not taking safety precautions places the subjects in danger. For instance, loops that form anywhere within the circuit induce current and possible heat injury. Acquisition with the electrodes at high impedance not only hampers the EEG data quality but also poses a potential hazard to the subject (thermal injury due to high current density). The same hazard applies to broken electrodes. Cables placed in close proximity to the MR bore wall, in other words, far from the center, also pose a potential heating hazard to the subject (heating due to antenna effect) ${ }^{25}$. This protocol emphasizes the following safety aspects: no loops form within the circuit between the subject and the amplifier, all electrodes have low impedance during the MRI scan, and all cables are placed in the center of the bore. Beginner operators are advised to undergo training and follow the manufacturer's guidelines found in the user manual and demonstration videos ${ }^{20}$ to avoid any safety concerns.

The major causes of artifacts found on EEG-fMRI are switching gradient of the MRI, BCG, or the subject's gross or subtle movements (face movements, clenching, swallowing etc.). In some MRI setups, artifacts caused by the helium pump and ventilators also significantly compromise the EEG signals. MR gradient artifacts are rather consistent in the waveforms and can be sufficiently corrected using a templatebased subtraction technique if they are fully recorded without distortion using amplifiers with a sufficient dynamic range $^{24}$. BCG artifacts are usually corrected using either the subtraction technique ${ }^{26}$, independent component analysis ${ }^{6}$, optimal basis set ${ }^{8}$, or a combination of these techniques ${ }^{10}$. Recently, artifact removal using simple regression based on signals acquired simultaneously with carbon wire loops has been developed ${ }^{7,9}$. The protocol presented here illustrates the technical aspect, with the aim of providing an introductory guide for those who are interested in using this method. 
This method removes BCG, subtle subject movements, and helium pump artifacts and the resulting EEG signals are reportedly superior to those corrected using other methods ${ }^{7,9}$. However, larger motion artifacts, especially those containing swaying movements, are not removable even using this method ${ }^{7}$. Despite the improvement of these artifact-removal methodologies over the years, inconsistent artifacts, including those caused by MRI machinery-induced vibration are still difficult to remove. Moreover, the more extensive the artifact removal procedure, the higher the risk of losing some real EEG signals. Therefore, good preparation that can minimize the inconsistent artifacts remains most important in EEG-fMRI acquisition. In this protocol, these artifacts are minimized by using: (1) an elastic bandage to wrap the head and memory foam pillows to immobilize the head in the head coil, to reduce possible vibration of the wires while maintaining the subject's comfort; (2) cotton and medical adhesive tape to reduce vibration of the ECG electrode wire that may not be fully immobilized by the subject's own weight (partially floating between the subject and the table especially in a thin subject); and (3) sandbags to immobilize the cables placed in the MRI bore. These are important techniques to minimize difficultto-removed MRI machinery-induced vibration artifacts, which have not been described in the previously published EEGfMRI protocol ${ }^{20}$. In that protocol, subjects were placed in the scanner without additional wrapping over the EEG cap and padding around the head, and cables were only taped at a few points without immobilization using sandbags. Based on 20 years of experience at the Montreal Neurological Institute, we realized that those measures may contribute to the susceptibility of the electrode wires and cables to MRI machinery-induced vibration, although they are rarely emphasized in most EEG-fMRI studies ${ }^{6}$. Minimizing the MRI machinery-induced vibration subsequently leads to better quality and readability of the EEG, which is particularly useful for identifying subtle changes or events in the $E E G^{6}$, such as small epileptic discharges in epilepsy studies and single-trial ERPs in neurocognitive studies.

The detection of ERPs in EEG signals is a prerequisite for cognitive neuroscience studies. In contrast to the classic grand average response across trials, ERP single-trial detection, which provides insights into brain dynamics in response to a particular stimulus, is becoming a new target in modern cognitive neuroscience studies and noninvasive brain-computer interface research ${ }^{27}$. Application of the present protocol may contribute to increasing efficiency in these research fields.

The protocol is best suited for the MRI-compatible EEG system used in this study. Nevertheless, we believe that the important points may also be applicable to other MRIcompatible EEG systems.

\section{Disclosures}

The authors report no disclosures relevant to the manuscript.

\section{Acknowledgments}

This study was sponsored by the National Institute of Information and Communications Technology of Japan (NICT).

The authors thank the MRI physicists and technologists at the Center for Information and Neural Networks for their dedication in acquiring good quality MRI data.

Dr. Khoo is funded by Grant-in-Aid for Scientific Research (Nos. 18H06261, 19K21353, 20K09368) from the Ministry of Education, Culture, Sports, Science, and Technology of Japan and a grant by the National Institute of Information 
and Communications Technology of Japan (NICT), and was supported by Mark Rayport and Shirley Ferguson Rayport fellowship in epilepsy surgery and the Preston Robb fellowship of the Montreal Neurological Institute (Canada), a research fellowship of the Uehara Memorial Foundation (Japan). She received a sponsored award from the Japanese Epilepsy Society, support from the American Epilepsy Society (AES) Fellows program, and travel bursary from the International League Against Epilepsy (ILAE).

Dr. Tani is funded by Grant-in-Aid for Scientific Research (No. 17K10895) from the Ministry of Education, Culture, Sports, Science, and Technology of Japan and received research support from Mitsui-Kousei Foundation, funding for a trip from Medtronic, royalties from the publication of articles (Gakken Medical Shujunsha, Igaku-shoin), and honoraria from serving as speaker (Medtronic, Daiichi-Sankyo Pharmaceuticals, Eisai Pharmaceuticals).

Dr. Oshino is funded by Grant-in-Aid for Scientific Research (No. 17K10894) from the Ministry of Education, Culture, Sports, Science, and Technology of Japan. He received royalties from the publication of articles (Medicalview, Igakushoin), and honoraria from serving as speaker (Insightec, Eisai Pharmaceuticals, Daiichi-Sankyo Pharmaceuticals, UCB, Otsuka Pharmaceuticals, Teijin Pharma, Yamasa Corporation).

Dr. Fujita is funded by Grant-in-Aid for Scientific Research (No. 19K18388) from the Ministry of Education, Culture, Sports, Science, and Technology of Japan.

Dr. Gotman is funded by the Canadian Institutes of Health Research (No. FDN 143208).

Dr. Kishima is funded by Grant-in-Aid for Scientific Research (Nos. 18H04085, 18H05522, 16K10212, 16K10786) from the Ministry of Education, Culture, Sports, Science, and Technology of Japan, Cross-ministerial Strategic Innovation Promotion Program (No. SIPAIH18E01), Japan Agency for Medical Research and Development, and Japan Epilepsy Research Foundation.

\section{References}

1. Krakow, K. et al. EEG-triggered functional MRI of interictal epileptiform activity in patients with partial seizures. Brain. 122, 1679-1688 (1999).

2. Ives, J. R., Warach, S., Schmitt, F., Edelman, R. R., Schomer, D. L. Monitoring the patient's EEG during echo planar MRI. Electroencephalography and Clinical Neurophysiology. 87 (6), 417-420 (1993).

3. Nunez, P. L., Silberstein, R. B. On the relationship of synaptic activity to macroscopic measurements: does co-registration of EEG with fMRI make sense? Brain Topography. 13 (2), 79-96 (2000).

4. Kruggel, F., Wiggins, C. J., Herrmann, C. S., von Cramon, D. Y. Recording of the event-related potentials during functional MRI at 3.0 Tesla field strength. Magnetic Resonance in Medicine. 44 (2), 277-282 (2000).

5. Lemieux, L., Allen, P. J., Franconi, F., Symms, M. R., Fish, D. R. Recording of EEG during fMRI experiments: patient safety. Magnetic Resonance in Medicine. 38 (6), 943-952 (1997).

6. Benar, C. et al. Quality of EEG in simultaneous EEGfMRI for epilepsy. Clinical Neurophysiology. 114 (3), 569-580 (2003).

7. Masterton, R. A., Abbott, D. F., Fleming, S. W., Jackson, G. D. Measurement and reduction of motion and 
ballistocardiogram artefacts from simultaneous EEG and fMRI recordings. Neuroimage. 37 (1), 202-211 (2007).

8. Niazy, R. K., Beckmann, C. F., lannetti, G. D., Brady, J. M., Smith, S. M. Removal of FMRI environment artifacts from EEG data using optimal basis sets. Neuroimage. 28 (3), 720-737 (2005).

9. van der Meer, J. N. et al. Carbon-wire loop based artifact correction outperforms post-processing EEG/fMRI corrections--A validation of a real-time simultaneous EEG/fMRI correction method. Neuroimage. 125, 880-894 (2016).

10. Debener, S. et al. Improved quality of auditory eventrelated potentials recorded simultaneously with 3-T fMRI: removal of the ballistocardiogram artefact. Neuroimage. 34 (2), 587-597 (2007).

11. Mele, G. et al. Simultaneous EEG-fMRI for functional neurological assessment. Frontiers in Neurology. 10, 848 (2019).

12. Gotman, J., Kobayashi, E., Bagshaw, A. P., Benar, C. G., Dubeau, F. Combining EEG and fMRI: a multimodal tool for epilepsy research. Journal of Magnetic Resonance Imaging. 23 (6), 906-920 (2006).

13. Ford, J. M., Roach, B. J., Palzes, V. A., Mathalon, D. $H$. Using concurrent EEG and $\mathrm{fMRI}$ to probe the state of the brain in schizophrenia. Neurolmage: Clinical. 12, 429-441 (2016).

14. Menon, V., Crottaz-Herbette, S. in International Review of Neurobiology. Elsevier. 291-321 (2005).

15. Liu, T. T. Efficiency, power, and entropy in eventrelated fMRI with multiple trial types. Part II: design of experiments. Neuroimage. 21 (1), 401-413 (2004).
16. Liu, T. T., Frank, L. R. Efficiency, power, and entropy in event-related FMRI with multiple trial types. Part I: theory. Neuroimage. 21 (1), 387-400 (2004).

17. Gotman, J., Benar, C. G., Dubeau, F. Combining EEG and FMRI in epilepsy: methodological challenges and clinical results. Journal of Clinical Neurophysiology. 21 (4), 229-240 (2004).

18. Gutberlet, I. in Simultaneous EEG and fMRI. eds M. Ullsperger, S. Debener). Oxford University Press. Ch. 2.1, 69-84 (2010).

19. Operating and Reference Manual for use in a laboratory and MR environment. in BrainAmp series \& BrainAmp MR series. Brain Products GmbH. Ch. 2 (2020).

20. Mullinger, K. J., Castellone, P., Bowtell, R. Best current practice for obtaining high quality EEG data during simultaneous FMRI. Journal of Visualized Experiments: JoVE. (76), 50283 (2013).

21. Ragazzoni, A. et al. "Hit the missing stimulus". A simultaneous EEG-fMRI study to localize the generators of endogenous ERPs in an omitted target paradigm. Scientific Reports. 9 (1), 3684 (2019).

22. Faulkner, W. New MRI Safety Labels \& Devices., <https://www.ismrm.org/smrt/ESignals/2016FEBRUARY/eSig_5_1_hot_2.htm> (2016).

23. Conditions for safe use of BrainAmp MR amplifiers and accessories in the MR environment. in Performing simultaneous EEG-fMRI measurements. Brain Products GmbH. Ch. 7, 26-32 (2020).

24. Allen, P. J., Josephs, O., Turner, R. A method for removing imaging artifact from continuous EEG recorded during functional MRI. Neuroimage. 12 (2), 230-239 (2000). 
25. Dempsey, M. F., Condon, B. Thermal injuries associated with MRI. Clinical Radiology. 56 (6), 457-465 (2001).

26. Allen, P. J., Polizzi, G., Krakow, K., Fish, D. R., Lemieux, L. Identification of EEG events in the MR scanner: the problem of pulse artifact and a method for its subtraction. Neuroimage. 8 (3), 229-239 (1998).

27. Cecotti, H., Ries, A. J. Best practice for singletrial detection of event-related potentials: Application to brain-computer interfaces. International Journal of Psychophysiology. 111, 156-169 (2017). 